\title{
INTRODUCTION TO THE SPECIAL ISSUE ON MOBILE CLOUD APPLICATIONS AND CHALLENGES
}

We are happy to present this special issue of thee Scientific Journal Scalable Computing: Practice and Experience. In this special issue on Mobile Cloud Applications and Challenges (Volume 19 No 4 December 2018), we have selected five papers, which gone through a peer review according to the journal policy. All the papers represents novel results in the field of Mobile and Cloud Applications.

The first paper presents overview of mobile cloud computing, cloudlet technology, security and privacy issues and limitations of mobile cloud commuting. The second paper presents a spatial partition, global index and map reduce operation were studied. The trail results that the proposed indexing cloud framework performs improved results. The third paper proposes an authentication model along with data security in a public cloud storage environment which successful detects the unauthenticated access or any anomaly in data. The fourth paper analyses the student success ratio which uses a cloud based technology to implement and design SaaS. Graph based complex network are used for analysing the course. The fifth paper presents Overlapping community detection in social networks. The proposed algorithm uses parallel processing engine to resolve the delay problem.

We use this opportunity to thank all the contributors to this special issue. We would like to express our special gratitude for the Editor-in-chief, Professor Dana Petcu for her constant support for carrying this special issue.

Rajkumar Rajasekaran, Vellore Institute of Technology, India 